\title{
A Note about Bezdek's Conjecture on Covering an Annulus by Strips
}

\author{
Yuqin Zhang ${ }^{1 *}$ and Ren Ding ${ }^{2}$ \\ ${ }^{1}$ Department of Mathematics, Tianjin Univerity \\ Tianjin 300072, China \\ yqinzhang@163.com yuqinzhang@126.com \\ 2 College of Mathematics, Hebei Normal University \\ Shijiazhuang 050016, China \\ rending@hebtu.edu.cn rending@heinfo.net
}

Submitted: May 19, 2007; Accepted: Jun 3, 2008; Published: Jun 13, 2008

Mathematics Subject Classifications: 52C15

\begin{abstract}
A closed plane region between two parallel lines is called a strip. András Bezdek posed the following conjecture: For each convex region $K$ there is an $\varepsilon>0$ such that if $\varepsilon K$ lies in the interior of $K$ and the annulus $K \backslash \varepsilon K$ is covered by finitely many strips, then the sum of the widths of the strips must be at least the minimal width of $K$. In this paper, we consider problems which are related to the conjecture.
\end{abstract}

\section{$1 \quad$ Introduction and Basic Definitions}

A closed plane region between two parallel lines at distance $d$ is called a strip of width $d$. For each direction $\theta, 0 \leq \theta \leq \pi$, a convex region $M$ has two parallel supporting lines and the distance between them is denoted by $\omega(\theta)$. The minimum $\omega(\theta)$ is called the minimal width of $M$. In the case of a triangle, the minimal width is the altitude on the longest side.

Let $O$ denote the origin of the plane $E^{2}$. For a given convex set $K$ and $\varepsilon>0$, let $\varepsilon K$ denote a homothetic copy of $K$ consisting of all points $X$ such that $\overrightarrow{O X}=\varepsilon \overrightarrow{O Y}$, where $Y \in K$.

Tarski [5] conjectured and Bang [1] proved that if a convex region $K$ can be covered by a finite collection of strips, then the sum of the widths of the strips must be at least

\footnotetext{
${ }^{*}$ This research was supported by National Natural Science Foundation of China (10571042, 10701033, 10671014).
} 
the minimal width of $K$. András Bezdek [2] posed the following conjecture and proved two theorems:

Conjecture. ([2]) For each convex region $K$ there is an $\varepsilon>0$ such that if $\varepsilon K$ lies in the interior of $K$ and the annulus $K \backslash \varepsilon K$ is covered by finitely many strips, then the sum of the widths of the strips must be at least the minimal width of $K$.

Theorem A. ([2]) Let $C$ be the unit square and let $\varepsilon$ be equal to $1-1 / \sqrt{2} \approx 0.29$. If $\varepsilon C$ lies in the interior of the square $C$ and the annulus $C \backslash \varepsilon C$ is covered by finitely many strips, then the sum of the widths is at least 1 .

Theorem B. ([2]) The conjecture is true for each polygon whose incircle is tangent to two of its parallel sides. In particular, it is true for regular polygons with an even number of sides.

White and Wisewell obtained in 2007 the following result:

Theorem C. ([6]) Let $P$ be a convex polygon. If there is a minimal-width chord of $P$ that meets a vertex and divides the angle at that vertex into two acute angles, then for every $\varepsilon>0$ an $\varepsilon$-scaled copy of $P$ can be removed so that the resulting annulus can be covered by strips of total width strictly less than the minimal width of $P$.

We would like to make the following remarks here. In the previous version of this article which is in the References of the paper [6] by White and Wisewell, we claimed the following result as a counter example to Bezdek's conjecture:

Proposition The conjecture is not true for any equilateral triangle.

Clearly this proposition is a special case of Theorem $\mathrm{C}([6])$. But our three-sentence proof is quite elementary and independent of [6]. Maybe it's worthy of consideration. Here is the proof.

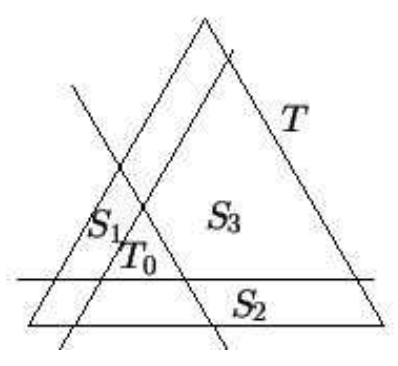

Figure 1:

Proof. It's an elementary fact that the sum of the distances from any point in an equilateral triangle $T$ to its three sides is equal to the minimal width of $T$. Let the width of $T$ be $\omega$ and $T_{0}=\varepsilon T \subset T$, then the annulus $T \backslash T_{0}$ is covered by the strips $S_{1}, S_{2}, S_{3}$, as shown in Fig.1. But the sum of the three strips' widths is equal to $(1-\varepsilon) \omega$ which is strictly less than $\omega$. 


\section{$2 \quad$ Main Results}

In this paper we consider more problems which are related to the conjecture. We need a few lemmas for our further discussion.

Lemma 1. ([2],[3]) Let $S_{1}, S_{2}, \cdots, S_{n}$ be a finite number of strips such that $S_{i}$ is of width $d_{i}$. Let $M$ be a centrally symmetric convex polygon of $2 n$ sides such that each pair of opposite sides are of length $d_{i}$ and perpendicular to one of the strips $S_{i}$. Assume that the origin $O$ is the center of $M$. Denote by $u_{i}$ the vector which is perpendicular to $S_{i}$ and has magnitude $\frac{1}{2} d_{i}$. Then one of the $2 n$ points, say $P$, of the form $\varepsilon_{1} u_{1}+\varepsilon_{2} u_{2}+\varepsilon_{n} u_{n}$ (where each $\varepsilon_{i}$ can be \pm 1$)$ does not belong to the interior of any strip $S_{i}(i=1, \cdots, n)$.

Moreover, if no two of the strips are parallel and no three of the boundary lines pass through the same point, then the strips cannot cover a neighborhood of the point $P$ and thus they cannot cover a neighborhood of $M$.

Lemma 2. [4] Let $M$ be a centrally symmetric convex polygon with perimeter $l . \triangle A B C$ is a circumscribed triangle of $M$, then the minimal width $w$ of $\triangle A B C$ is not greater than $\frac{l}{2}$.

Theorem 1. Let $T$ be a triangle with minimal width $w$ and $S_{1}, S_{2}, \cdots, S_{n}$ be $n$ strips such that $S_{i}$ is of width $d_{i}$ and $\sum_{i=1}^{n} d_{i}=d$. If $d<w$, for any $\varepsilon$ with $0<\varepsilon<1-(d / w)$, $\varepsilon T$ lies in the interior of $T$, then the annulus $T \backslash \varepsilon T$ can not be covered by the $n$ strips.

Proof. Without loss of generality, let $T=\triangle A B C$ with $|B C| \geq|C A| \geq|A B|$ and $T_{0}=\varepsilon T$. Assume that $B C$ is horizontal. Obviously, the minimal width $w$ of $T$ is the altitude on $B C$.

Assume that the $n$ strips are in general position, otherwise, we expand the covered parts by moving the strips slightly so that the sum of the widths is still less than $w$, while the strips are in general position. Consider the centrally symmetric convex polygon $M$ of $2 n$ sides which corresponds to the $n$ strips as in Lemma 1, that is, each pair of opposite sides of $M$ are perpendicular to one of the strips $S_{i}$ and of length $d_{i}$. It's easy to see that for any given triangle $T$, each convex region $M$ has a circumscribed triangle $T_{1}$ similar to $T$. So let $T_{1}=\triangle A_{1} B_{1} C_{1}$ be the circumscribed triangle of $M$ with $B_{1} C_{1}$ horizontal and $T_{1}$ be similar to $T$. By Lemma $2, w_{1}=w\left(T_{1}\right) \leq \frac{l}{2}=\frac{1}{2}\left(2 \sum_{i=1}^{n} d_{i}\right)=d<w$. As $T$ is a triangle with minimal width $w, \triangle A B C \simeq \triangle A_{1} B_{1} C_{1}$, we can translate $T_{1}$ with the inscribed polygon $M$ until the angles $\angle B A C$ and $\angle B_{1} A_{1} C_{1}$ coincide, and it follows that $T_{1}$ lies in $T$ and $B C \| B_{1} C_{1}$. Now denote the distance between $B_{1} C_{1}$ and $B C$ by $\varepsilon_{1}=w-w_{1} \geq w-d$. Take a positive number $\varepsilon$ which is slightly less than $1-d / w$. Then add an additional strip $S_{n+1}$ which is horizontal with width $d_{n+1}$ satisfying $\varepsilon_{1}>d_{n+1}>\varepsilon$. If $M$ has a pair of horizontal sides, then we choose a direction sufficiently close to horizontal. So the inner triangle $T_{0}$ can be covered by $S_{n+1}$ while the strips $S_{1}, S_{2}, \cdots, S_{n+1}$ remain in general position.

Denote by $M_{1}$ the convex polygon corresponding to the given $n+1$ strips. A pair of vertical sides of length smaller than $\varepsilon_{1}$ are added to the sides of $M$ to get the polygon 
$M_{1}$. Thus $M_{1}$ can also be translated into the interior of $T$. According to Lemma $1, M_{1}$ can not be covered by $S_{1}, S_{2}, \cdots, S_{n+1} . M_{1} \subseteq T, T_{0}$ is covered by $S_{n+1}$, and so $T \backslash T_{0}$ can not be covered by $S_{1}, S_{2}, \cdots, S_{n}$.

We denote by $c(\varepsilon K)$ any copy of $\varepsilon K$ obtained by translating or rotating $\varepsilon K$, and if $c(\varepsilon K) \subset K, K \backslash c(\varepsilon K)$ is also called an annulus. Denote by $P(u, v, \beta)$ a parallelogram with two adjacent sides $u, v(u \leq v)$ and the smaller angle $\beta$.

For the proof of our third result, we need another three lemmas besides Lemma 1:

Lemma 3. Let $M$ be a centrally symmetric convex polygon with perimeter $l$. If $P(u, v, \beta)$ is a circumscribed parallelogram of $M$, then $u \leq \frac{l}{2 \sin \beta}, v \leq \frac{l}{2 \sin \beta}$.

Proof. It's easy to get a circumscribed parallelogram $A B C D$ of $M$ with $|A B|=|C D|=$ $u,|D A|=|B C|=v$ and smaller angle $\angle A B C$ equal to $\beta$. Let $E$ be the common point of the side $A B$ and $M$, and $F$ be the common point of the side $C D$ and $M$. Then it's obvious that $|E F|<\frac{l}{2}$ and $v \sin \beta \leq|E F|$, so $v \leq \frac{l}{2 \sin \beta}$. In the same way, we get $u \leq \frac{l}{2 \sin \beta}$.

By the law of sines, we have:

Lemma 4. Among all the triangles with a side and its opposite angle given, the isosceles one has the largest perimeter.

Lemma 5. Let parallelogram $P=P(u, v, \beta)$ be the circumscribed parallelogram of a quadrilateral EFGH whose perimeter is less than $l$, then the perimeter of $P$ is less than $\sqrt{\frac{2}{1-\cos \beta}} l$.

Proof. Let $A, B, C, D$ be the four vertices of the parallelogram $P(u, v, \beta)$, and $E, F, G, H$ lie on $A B, B C, C D, D A$ respectively. If $|A H|=|A E|=a_{1}$, by the law of cosines, we have $2 a_{1}^{2}+2 a_{1}^{2} \cos \beta=|E H|^{2}$, hence $a_{1}=\sqrt{\frac{1}{2(1+\cos \beta)}}|E H|<\sqrt{\frac{1}{2(1-\cos \beta)}}|E H|$. For the given $|E H|$ and $\pi-\beta$, by Lemma $4,|A H|+|A E| \leq 2 a_{1}<\sqrt{\frac{2}{1-\cos \beta}}|E H|$. Similarly we obtain $|B E|+|B F|<\sqrt{\frac{2}{1-\cos \beta}}|E F|,|F C|+|C G|<\sqrt{\frac{2}{1+\cos \beta}}|F G|<\sqrt{\frac{2}{1-\cos \beta}}|F G|$, $|H D|+|D G|<\sqrt{\frac{2}{1-\cos \beta}}|H G|$. So $p(A B C D)<\sqrt{\frac{2}{1-\cos \beta}} p(E F G H)<\sqrt{\frac{2}{1-\cos \beta}} l$.

On the basis of Theorem A [2], we obtain the following result which may be considered as a generalization in some sense.

Theorem 2. For a given parallelogram $P=P(u, v, \beta)$, assume that $\varepsilon=\left(1-\sqrt{\frac{1+\cos \beta}{2}}\right) u$ and $P_{0}=c(\varepsilon P)$ is any copy of $\varepsilon P$ such that the angle from the longer side of $P$ to the perpendicular line of the longer side of $P_{0}$ is $\beta$ (see Fig.2). If $P_{0}$ lies in the interior of $P$ and the annulus $P \backslash P_{0}$ is covered by finitely many strips, then the sum of the width of strips is at least $u \sin \beta$. 

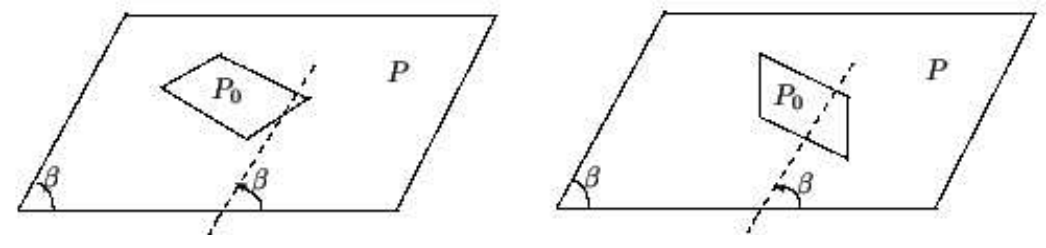

Figure 2:

Proof. Let $S_{1}, S_{2}, \cdots, S_{n}$ be the strips with widths $d_{1}, d_{2}, \cdots, d_{n}$ which cover $P \backslash P_{0}$. Assume that $P$ has a pair of horizontal sides. As in the proof of Theorem 1, assume that the strips are in general position. Suppose, on the contrary, that $d_{1}+d_{2}+\cdots+d_{n}<u \sin \beta$ and we show that the union of strips $S_{i}$ does not contain the annulus $P \backslash P_{0}$. Let $M$ be the centrally symmetric convex $2 n$-gon corresponding to the $n$ strips and satisfying that each pair of opposite sides are of length $d_{i}$ and perpendicular to one of the strips $S_{i}$. It's easy to get a circumscribed parallelogram $P_{1}=P_{1}\left(u_{1}, v_{1}, \beta\right)$ of $M$. Translate $P_{1}$ along with the inscribed polygon $M$ until the left upper vertices of the parallelograms $P$ and $P_{1}$ coincide. Since the perimeter of $M$ is less than $2 u \sin \beta$, by Lemma 3 , we have $u_{1} \leq \frac{2 u \sin \beta}{2 \sin \beta}=u, v_{1} \leq u \leq v$ and it follows that $P_{1}$ lies in $P$. Choose a vertex of $M$ on each side of $P$ and connect them counterclockwise. The quadrilateral obtained has a perimeter less than $2 u \sin \beta$ as well. By Lemma 5 , the perimeter of $P_{1}$ is less than $2 u \sin \beta \sqrt{\frac{2}{1-\cos \beta}}$. So one of the sides of $P_{1}$ is less than $\frac{u \sin \beta \sqrt{\frac{2}{1-\cos \beta}}}{2}=u \sqrt{\frac{1+\cos \beta}{2}}$. Without loss of generality, assume that $u_{1}<u \sqrt{\frac{1+\cos \beta}{2}}$. Add an additional strip $S_{n+1}$ such that

1. The angle from the horizontal side of $P$ to the boundary line of $S_{n+1}$ is $\frac{\pi}{2}+\beta$;

2. Its width $d_{n+1}$ satisfies $\left(u-u_{1}\right) \sin \beta>d_{n+1}>\left(1-\sqrt{\frac{1+\cos \beta}{2}}\right) u \sin \beta$;

3. It covers the inner parallelogram $P_{0}$ of the annulus, while the strips $S_{1}, S_{2}, \cdots$, $S_{n+1}$ remain in general position.

Denote by $M_{1}$ the convex $2(n+1)$-polygon corresponding to the given $n+1$ strips. A pair of sides of length less than $\left(u-u_{1}\right) \sin \beta$ and parallel to the shorter sides of $P$ are added to the sides of $M$ to get the polygon $M_{1}$. Thus $M_{1}$ can be translated into the interior of $P$ as well. According to Lemma $1, M_{1}$ can not be covered by $S_{1}, S_{2}, \cdots, S_{n+1}$. $M_{1} \subseteq P, P_{0}$ is covered by $S_{n+1}$, and so $P \backslash P_{0}$ can not be covered by $S_{1}, S_{2}, \cdots, S_{n}$, a contradiction. So $\sum_{i=1}^{n} d_{i} \geq u \sin \beta$.

\section{Acknowledgements}

We are grateful to the anonymous referees for their expert comments and advice. 


\section{References}

[1] T. Bang, A solution of the plank problem, Proc.Amer.Math.Soc., 2 (1951), 990-993.

[2] A. Bezdek, Covering an annulus by strips, Discrete Comput. Geom., 30 (2003), 177180.

[3] H.G. Eggleston, On triangles circumscribing plane convex sets, J. London Math.Soc., 28(1953), 36-46.

[4] Zun Shan, Combinatorical Geometry (in Chinese), Shanghai Educational Press, 1995.

[5] A. Tarski, Uwagi o stopniu rownowaznosci, Odbilka Z. Parametru, 2 (1932), 310-314.

[6] S. White and L. Wisewell, Covering polygonal annuli by strip, Discrete Comput. Geom. 37 (2007), 577-585. 